\title{
SEPARATION THEOREMS FOR NONCONVEX SETS IN SPACES WITH NON-SYMMETRIC SEMINORM
}

\author{
G. E. IVANOV AND M. S. LOPUSHANSKI
}

Abstract. The theory of weakly convex sets in Banach spaces with non-symmetric seminorm is developed. The separation theorem with sphere or (in a general case) with the boundary of a shifted quasiball for two closed disjoint subsets of a Banach space, one of which is prox-regular or weakly convex, and the other is the summand of a ball or quasiball is proven.

Mathematics subject classification (2010): 41A50, 41A65, 52A21.

Keywords and phrases: Weakly convex sets, prox-regular sets, quasiball, non-symmetric seminorm.

\section{REFERENCES}

[1] M. V. BALASHOV AND G. E. IVANOv, Weakly convex and proximally smooth sets in Banach spaces, Izvestiya Math. 73, 3 (2009), 455-499.

[2] F. Bernard, L. Thibault, N. Zlateva, Characterization of proximal regular sets in super reflexive Banach spaces, J. Convex Analysis 13, 3, 4 (2006), 525-559.

[3] G. Bouligand, Sur les surfaces dépourvues de points hyperlimites, Ann. Soc. Polon. Math. 9, (1930), 32-41.

[4] F. H. Clarke, R. J. Stern and P. R. Wolenski, Proximal smoothness and lower-C ${ }^{2}$ property, J. Convex Anal. 2, 1, 2 (1995), 117-144.

[5] J. A. Clarkson, Uniformly convex spaces, Trans. Amer. Math. Soc. 40, 3 (1936), 396-414.

[6] M. M. DAY, Some more uniformly convex spaces, Bull. Amer. Math. Soc. 47, 6 (1941), 504-507.

[7] H. Federer, Curvature measures, Trans. Amer. Math. Soc. 93, 63 (1959), 418-491.

[8] G. E. Ivanov, Weakly convex sets and functions: theory and applications, (in Russian) Fizmatlit, Moscow, 2006.

[9] G. E. IVANOv, Weak convexity of sets and functions in a Banach space, J. Convex Anal. 22, 62 (2015), 365-398.

[10] G. E. Ivanov, On well posed best approximation problems for a nonsymmetric seminorm, J. Convex Anal. 20, 62 (2013), 501-529.

[11] G. E. IVANOV AND M. S. LopUSHANSKI, Well-posedness of approximation and optimization problems for weakly convex sets and functions, Journal of Mathematical Sciences 209, 61 (2015), 66-87.

[12] R. A. Poliquin And R. T. RockAfellar, Prox-regular functions in variational analysis, Trans. Amer. Math. Soc. 348, 65 (1996), 1805-1838. 